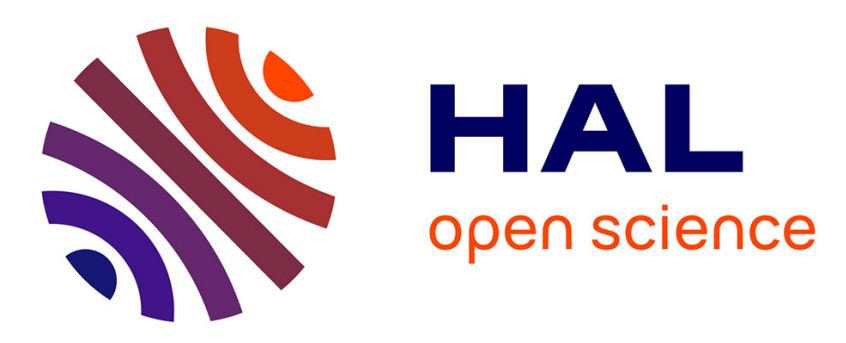

\title{
Uncertainty-driven Efficiently-Sampled Sparse Graphical Models for Concurrent Tumor Segmentation and Atlas Registration
}

Sarah Parisot, William Wells Iii, Stéphane Chemouny, Hugues Duffau, Nikos Paragios

\section{To cite this version:}

Sarah Parisot, William Wells Iii, Stéphane Chemouny, Hugues Duffau, Nikos Paragios. Uncertaintydriven Efficiently-Sampled Sparse Graphical Models for Concurrent Tumor Segmentation and Atlas Registration. ICCV - 14th International Conference on Computer Vision, Dec 2013, Sydney, Australia. hal-00858696

\section{HAL Id: hal-00858696 https://hal.inria.fr/hal-00858696}

Submitted on 5 Sep 2013

HAL is a multi-disciplinary open access archive for the deposit and dissemination of scientific research documents, whether they are published or not. The documents may come from teaching and research institutions in France or abroad, or from public or private research centers.
L'archive ouverte pluridisciplinaire HAL, est destinée au dépôt et à la diffusion de documents scientifiques de niveau recherche, publiés ou non, émanant des établissements d'enseignement et de recherche français ou étrangers, des laboratoires publics ou privés. 


\title{
Uncertainty-driven Efficiently-Sampled Sparse Graphical Models for Concurrent Tumor Segmentation and Atlas Registration
}

\author{
Sarah Parisot ${ }^{1,2,4}$ *, William Wells III ${ }^{3}$, Stéphane Chemouny ${ }^{4}$, Hugues Duffau ${ }^{5}$, Nikos Paragios $^{1,2}$ \\ ${ }^{1}$ Center for Visual Computing, Ecole Centrale de Paris, France \\ ${ }^{2}$ Equipe Galen, INRIA Saclay-Ile-de-France, France \\ ${ }^{3}$ Surgical Planning Laboratory, Brigham and Women's hospital, Harvard Medical School, USA \\ ${ }^{4}$ Intrasense SAS, Montpellier, France \\ ${ }^{5}$ Département de Neurochirurgie, Hopital Gui de Chauliac, CHU Montpellier, France
}

\begin{abstract}
Graph-based methods have become popular in recent years and have successfully addressed tasks like segmentation and deformable registration. Their main strength is optimality of the obtained solution while their main limitation is the lack of precision due to the grid-like representations and the discrete nature of the quantized search space. In this paper we introduce a novel approach for combined segmentation/registration of brain tumors that adapts graph and sampling resolution according to the image content. To this end we estimate the segmentation and registration marginals towards adaptive graph resolution and intelligent definition of the search space. This information is considered in a hierarchical framework where uncertainties are propagated in a natural manner. State of the art results in the joint segmentation/registration of brain images with low-grade gliomas demonstrate the potential of our approach.
\end{abstract}

\section{Introduction}

Combined atlas based tumor segmentation and registration is an active research field. Most of the existing methods treat the problems sequentially [1, 12] or alternatively through an EM-like approach [6]. In [11] a discrete formulation was proposed for one-shot atlas-based registration and segmentation of brain tumors. This method couples the two problems and seeks their common solution. Despite the theoretical elegance of such a method, it inherits the major short-comings of discrete methods, that is lack of precision due the (i) requirement of a small label set and therefore sparse quantization of the search space, (ii) the constraints

*This work was supported by ANRT (grant 147/2010), Intrasense and the European Research Council Starting Grant Diocles (ERC-STG259112). on the form of the graph structure, the number of nodes and their connectivity in order to keep the computational burden manageable.

Despite the enormous amount of work in segmentation and registration, estimating the uncertainty in the result has little prior work. This is mostly the case due to the complexity of the solution space of these problems. MonteCarlo sampling among light variants like multiple hypotheses testing [3] were considered to certain extend in biomedical image analysis and computer vision. Early work on point-based registration using the iterate closed point algorithm $[13,16]$ have associated the contribution of the samples in the matching process according to their matching uncertainty. This idea was further explored in the context of segmentation by deformation using implicit shape representations [17] of brain structures as well as more recently in [7] where a statistical interpretation was proposed for voxellike decisions. Graph-theoretical formulations offer efficient means of measuring uncertainties of the obtained solutions [8]. These ideas were explored in [5] towards adaptive sampling of the label space according to the directional uncertainties of the current solution. The proposed strategy was able to improve the quality of the solution of discrete methods due to the intelligent sampling. On the other hand, it failed to use this information for adaptive refinement of the graph structure, a task that becomes a necessity when considering combined segmentation and registration as in [11] due to the fact that voxel based segmentation labels are difficult to express with a limited graph structure, and registration becomes problematic either in areas with discontinuities or at the borders between tumors and normal tissues.

In this paper we propose a novel graphical model that is endowed with a stochastic behavior. The most closely related work can be found in [5, 11] and in [15] where an alternative method to combine multiple-resolution grids 
in deformable registration was proposed towards contentdriven interpolation. The proposed hierarchical formulation exploits the form of the objective function to determine registration and segmentation uncertainties. They are then used to improve the precision of the sampling and determine the resolution of the graph at sub-sequent finer resolution levels. This is achieved with a hierarchical graph where nodes and decisions taken at coarse resolution level are linked and penalize deviations of decision for "dependent" nodes at the fine resolution level. The resulting formulation addresses the two problems in a stochastic manner and deals with one of the most important limitations of discrete methods, that is, adaptive graph partition of the domain and adaptive local quantization of the label space. The method was tested with promising results on the segmentation of low grade gliomas.

The remainder of this paper is organized as follows. In section 2 briefly reviews the concurrent segmentation and registration formulation of [11], while section 3 focuses on the estimation of uncertainties using the graph beliefs and their propagation to an adaptive hierarchical construction of the combined graphical model. The implementation details and experimental validation are part of section 4 while the last section concludes the paper.

\section{Joint Registration and Segmentation Method}

Let us consider a target volume $\mathrm{V}$, a subject featuring a tumor, and a healthy atlas A, defined on the domain $\Omega$. In the task of image registration, we aim at finding a transformation $\mathcal{T}$ that will map the source image to the target image:

$$
V(\mathbf{x})=A(\mathcal{T}(\mathbf{x})))
$$

The presence of the tumor renders the registration more difficult since it has no correspondences in the atlas. To deal with this issue, we aim at identifying the tumor area concurrently to the registration, i.e., we seek to assign a label $s \in\{0,1\}$ to each voxel of the image, where 1 corresponds to tumor and 0 to background. We adopt a Free Form Deformation approach [14] where a uniformly spaced sparse grid $\mathcal{G} \subset \Omega$ is superimposed to the image. The deformation and segmentation will be evaluated on the grid's control points, then evaluated on the whole image domain by interpolation:

$$
\begin{aligned}
& \mathcal{T}(\mathbf{x})=\mathbf{x}+\sum_{p \in \mathcal{G}} \eta(\|\mathbf{x}-\mathbf{p}\|) \mathbf{d}_{\mathbf{p}} \\
& \mathcal{S}(\mathbf{x})=\sum_{p \in \mathcal{G}} \eta(\|\mathbf{x}-\mathbf{p}\|) s_{\mathbf{p}}
\end{aligned}
$$

where $\mathbf{d}_{\mathbf{p}}$ and $s_{\mathbf{p}}$ are node $\mathbf{p}$ 's displacement vector and segmentation label respectively, and $\eta($.$) is the projection func-$ tion defining the influence of each grid control point over the image's voxels.
In order to estimate the optimal segmentation map $\mathcal{S}(\mathbf{x})$ and deformation field $\mathcal{T}(\mathbf{x})$ in a one shot optimization, we adopt a discrete Markov Random Field (MRF) approach [11]. Let us consider a predefined discrete displacement set $\mathcal{D}=\left\{\mathbf{d}^{\mathbf{1}}, . . \mathbf{d}^{\mathbf{n}}\right\}$. To each grid node $\mathbf{p}$, we seek to assign a label $l_{p} \in \mathcal{L}=\{1, . ., 2 n\}$ that corresponds to assigning to $\mathbf{p}$ a pair segmentation/displacement $\left\{\mathbf{d}^{\mathbf{l}_{\mathbf{p}}}, s^{l_{p}}\right\} \in \mathcal{D} \times\{0,1\}$, where $d^{l_{p}}=d^{l_{p}-n}$ and $s^{l_{p}}=1$ if $l_{p}>n$.

The optimal labeling $\mathbf{l}=\left\{l_{p}, \mathbf{p} \in \mathcal{G}\right\}$ is estimated by minimizing the MRF energy:

$$
\begin{aligned}
& E_{\text {reg,seg }}(\mathbf{l})=\frac{1}{|\mathcal{G}|} \sum_{p \in \mathcal{G}} \alpha V_{p, s e g}\left(l_{p}\right)+(1-\alpha) V_{p, r e g}\left(l_{p}\right) \\
& +\sum_{p \in \mathcal{G}} \sum_{q \in \mathcal{N}(p)} \alpha V_{p q, s e g}\left(l_{p}, l_{q}\right)+(1-\alpha) \lambda V_{p q, r e g}\left(l_{p}, l_{q}\right)
\end{aligned}
$$

where $\alpha$ is a parameter that balances the registration and segmentation terms, and $\lambda$ is a constant parameter describing the influence of the registration smoothness term.

The pairwise terms' role is to ensure local smoothness of the segmentation and deformation fields and are defined as:

$$
\begin{aligned}
& V_{p q, r e g}\left(\mathbf{d}^{\mathbf{l}_{\mathbf{p}}}, \mathbf{d}^{\mathbf{l}_{\mathbf{q}}}\right)=\frac{\left(\mathbf{d}^{\mathbf{l} \mathbf{p}}-\mathbf{d}^{\mathbf{l} \mathbf{q}}\right)^{2}}{\|\mathbf{p}-\mathbf{q}\|} \\
& V_{p q, s e g}\left(s^{l_{p}}, s^{l_{q}}\right)=\frac{\left|s^{l_{p}}-s^{l_{q}}\right|}{\|\mathbf{p}-\mathbf{q}\|}
\end{aligned}
$$

We want to find the existing correspondences between the source and target images' structures in the background area (label $\left.s^{l_{p}}=0\right)$. For all the potential displacements in $\mathcal{D}$, we compute a similarity measure $\rho($.) between both images. In the tumor area, we cannot rely on this similarity measure due to the absence of correspondences between tumor and healthy tissue. The same constant $\operatorname{cost} C_{t}$ is used instead for all displacements.

$$
\begin{array}{r}
V_{p, r e g}\left(l_{p}\right)=\int_{\Omega} \bar{\eta}(\|\mathbf{x}-\mathbf{p}\|) \\
\left(\left(1-s^{l_{p}}\right) \rho\left(V(\mathbf{x}), A\left(\mathbf{x}+\mathbf{d}^{\mathbf{l}_{\mathbf{p}}}\right)\right)+s^{l_{p}} C_{t m}\right) d x
\end{array}
$$

Since the similarity measure is evaluated at the pixel level, we introduce the function $\bar{\eta}($.$) to back project the informa-$ tion on the grid's nodes.

The tumor $p_{t m}$ and background $p_{b g}$ probabilities have been evaluated a priori via boosting [4]. The segmentation term seeks the maximum posterior given the prior probabilities assuming independence between measurements:

$$
\begin{array}{r}
V_{p, s e g}\left(l_{p}\right)=\int_{\Omega} \bar{\eta}(\|\mathbf{x}-\mathbf{p}\|) \\
\left(-\log \left(p_{b g}\left(\mathbf{x}+\mathbf{d}^{\left.\mathbf{l}_{\mathbf{p}}\right)}\right)\left(1-s^{l_{p}}\right)-\log \left(p_{t m}\left(\mathbf{x}+\mathbf{d}^{\mathbf{l}_{\mathbf{p}}}\right)\right) s^{l_{p}}\right) d x\right.
\end{array}
$$

The optimal labeling can be efficiently evaluated using Fast-PD [9], an optimization method based on linear programming. 


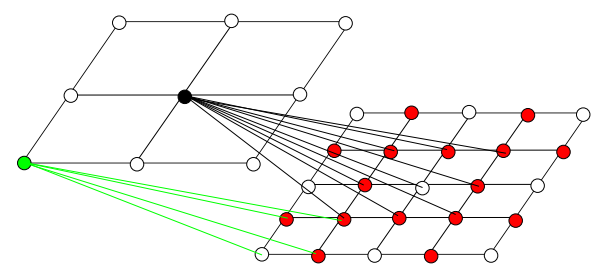

Figure 1: Visual representation of the grid refinement from level $j$ (left) to level j+1 (right). Grid resampling: the nodes that have direct correspondences appear in white, and the new nodes and edges are red. The edges connecting the 2 grids represent the nodes' neighborhood. The grid is shown in $2 D$ for increased visibility.

In [11] a compositional approach was considered where several MRF optimizations are performed at coarse to fine grid resolutions. At iteration $i$, the MRF energy is evaluated between the target image, and the deformed source image to recover the optimal labeling:

$$
l^{o p t, i}=\arg \min _{l} E_{\text {reg,seg }}\left(\mathbf{l} \mid V, A \circ \mathcal{T}^{i-1}\right)
$$

The new displacement field is then composed with the current displacement field $\mathcal{T}^{i-1}$ and the displacement set refined. Eventually, the grid's resolution is refined, and another series of optimizations are performed at a higher resolution.

Due to the joint registration and segmentation space, the computational complexity of the method increases quadratically at fine resolution levels, increasing running time while decreasing guarantees on the optimality of the obtained solution. In practice, the use of high-resolution grids (that is a necessity for precise segmentation) is prohibited, while at the same time sampling of the deformation space becomes an issue when seeking high resolution deformations. Both issues can be addressed using uncertainties towards sparse graphs in terms of structure and directional sampling of the deformation space.

\section{Uncertainty Driven Discrete Sampling}

We now describe the content driven grid construction and segmentation propagation. Consider a grid $\mathcal{G}_{j}$ of resolution $M \times N \times P$, a control point $\mathbf{c}_{\mathbf{j}} \in \mathcal{G}_{j}$., and $l_{c_{j}}^{\text {opt }}$ its optimal label recovered at iteration $i$. Our goal is to define the optimal displacement set for registration as well as the resolution of the next grid level $\mathcal{G}_{j+1}$ of maximal resolution $2 M-1 \times 2 N-1 \times 2 P-1$. We define an inter graph neighborhood system on the basis that each node $\mathbf{c}_{\mathbf{j}}$ has a direct spatial correspondence $\mathbf{p}_{\mathbf{j}+\mathbf{1}} \in \mathcal{G}_{j+1}$. The neighborhood $\mathcal{N}($.$) (shown in Fig.[1]) is defined by connecting \mathbf{c}_{\mathbf{j}}$ to $\mathbf{p}_{\mathbf{j}+\mathbf{1}}$ and its 26 immediate neighbors.

We rely on the min-marginal energies [8] that evaluate the minimum value of the energy under different con- straints. For our problem, we impose a non optimal label $k$ to the control point $\mathbf{c}_{\mathbf{j}}$ to evaluate the cost of a label swap:

$$
\Psi_{c_{j}, k, i}=\min _{l, l_{c_{j}}=k} E_{\text {reg, }, \text { seg }}\left(\mathbf{l} \mid V, A \circ \mathcal{T}^{i-1}\right)
$$

A label $k$ belonging to the same segmentation class $s^{k}=s^{l^{l_{j} p t}}$ represents a local perturbation from the optimal displacement. The directions towards which the energy weakly varies represent the local anisotropy and uncertainty of the registration. Indeed, a small variation of the energy suggest that the labels are almost as likely, while a strong increase in energy implies that the chosen label is certain in this direction. We compute each displacement's likelihood by marginalizing the max marginals over all possible displacements:

$$
P_{r e g}\left(l_{c_{j}}^{o p t}, k\right)=\frac{\exp \left(-\Psi_{c_{j}, k, i}\right)}{\sum_{l \in \mathcal{L}, s^{l}=s^{l_{c_{j}}^{o p t}}} \exp \left(-\Psi_{c_{j}, l, i}\right)}
$$

This approximates a 3D probability density function that can be assimilated to a Gaussian distribution. We adopt an intelligent displacement sampling based on the density's covariance scale and main axes, that indicate the directions where the uncertainty is maximum and a thorough sampling is necessary. Note that for the tumor label, and when the parameter $\alpha$ is low (dominant segmentation term), the local likelihoods are not of interest since the registration is mostly driven by the pairwise cost.

The next step is to define the grid sampling and segmentation propagation at the next resolution level. To this end, we call $\mathcal{G}_{j+1, \max }$ the maximum resolution at level $j+1$ and define a function $\mathcal{A}_{j+1}: \mathcal{G}_{j+1} \rightarrow\{0,1\}$, characterizing the node activation. The set of activated nodes $\left\{\mathbf{p} \in \mathcal{G}_{j+1, \text { max }}, \mathcal{A}_{j+1}=1\right\}$ will represent the actual resolution of $\mathcal{G}_{j+1}$. The activation function is defined as:

$\mathcal{A}_{j+1}(\mathbf{p})=\max \left(\sum_{\mathbf{c}_{\mathbf{j}} \in \mathcal{N}_{i}(\mathbf{p})} \delta\left(\mathbf{c}_{\mathbf{j}}, \mathbf{p}\right) \mathcal{A}_{j}\left(\mathbf{c}_{\mathbf{j}}\right), A_{r}(\mathbf{p}), A_{s}(\mathbf{p})\right)$

where $\delta$ is the Kronecker Delta function. The first term imposes that nodes in $\mathcal{G}_{j+1, \text { max }}$ that have a direct correspondent $\mathbf{c}_{\mathbf{j}} \in \mathcal{G}_{j, \max }$ (same spatial coordinates) are activated if their correspondent is $\left(\mathcal{A}_{j}\left(\mathbf{c}_{\mathbf{j}}\right)=1\right)$. The remaining nodes are either activated for segmentation $\left(A_{s}(\mathbf{p})=1\right)$ or registration $\left(A_{r}(\mathbf{p})=1\right)$. Given the strong interdependencies between segmentation and registration, a node activated for registration is also activated for segmentation and vice versa.

The registration activation is based on the local homogeneity of the region reachable by the node. The idea is that the min marginal energies will strongly vary if different structures are present in the region (intensity discontinuities), and remain relatively constant in an homogeneous 


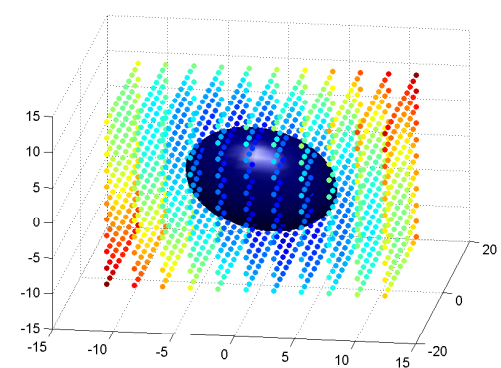

(a)

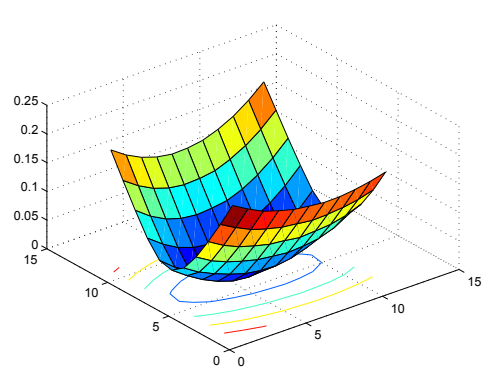

(b)

Figure 2: Registration uncertainty for one control point: (a) Min marginal values per displacement label (blue: low, red: high energy) and associated covariance matrix centered at the optimal label. (b) Min marginals visualization on a $2 D$ slice.

region. In the presence of discontinuities, precise displacements are necessary, while additional precision is not required in homogeneous regions.

For all nodes $\mathbf{c}_{\mathbf{j}} \in \mathcal{G}_{j}$, we compute the energy range over all displacement labels:

$$
R\left(\mathbf{c}_{\mathbf{j}}\right)=\max _{k \in \mathcal{L}, s^{k}=s^{l^{o p t}}} \Psi_{\mathbf{c}_{\mathbf{j}}, k, i}-\min _{k \in \mathcal{L}, s^{k}=s^{l^{o p p}}} \Psi_{\mathbf{c}_{\mathbf{j}}, k, i}
$$

We define the node activation criterion as follows:

$$
A_{r}(\mathbf{p})=H\left(\sum_{\mathbf{c}_{\mathbf{j}} \in \mathcal{N}_{i}(\mathbf{p})} \frac{1}{N} R\left(\mathbf{c}_{\mathbf{j}}\right)-\mu\right)
$$

where $H($.$) is the heaviside function, N$ is the number of nodes in $\mathcal{N}(\mathbf{p})$ and $\mu$ is the mean value of the min marginals range over all nodes in $\mathcal{G}_{j}$. A node in $\mathcal{G}_{j+1}$ will be activated if the mean energy range among its neighbors is higher than the mean range over all nodes, i.e. if the majority of its neighbors is close to a structure's boundary.

Similarly, the node activation for segmentation is guided by the min marginals. We rely on the segmentation uncertainty, computed by evaluating the global energy variation when the segmentation label changes. We compute the segmentation likelihood for a segmentation label $\mathrm{S}$ by

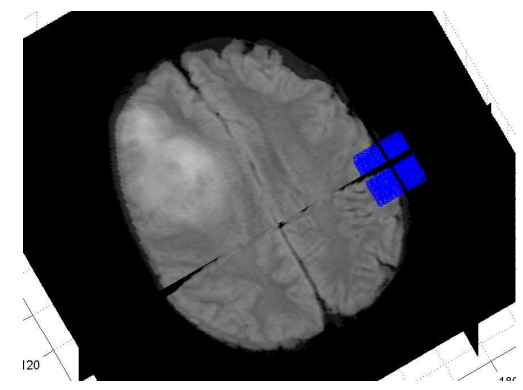

(a)

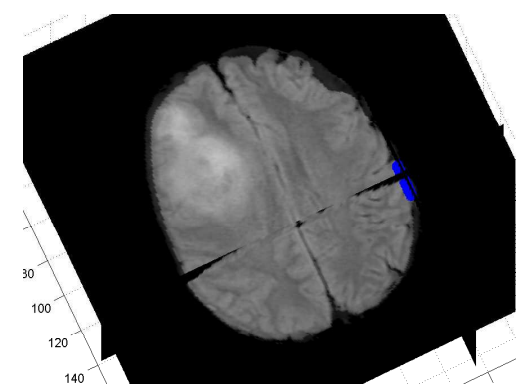

(b)

Figure 3: Displacement set resampling for one control point: (a) Original isotropic displacement set. (b) Uncertainty driven displacement set, following the brain boundaries.

marginalizing the max-marginals over all labels:

$$
P_{\text {seg }}\left(\mathbf{c}_{\mathbf{j}} \mid S\right)=\frac{\sum_{l \in \mathcal{L}, s^{l}=S} \exp \left(-\Psi_{\mathbf{c}_{\mathbf{j}}, l, t}\right)}{\sum_{l \in \mathcal{L}} \exp \left(-\Psi_{\mathbf{c}_{\mathbf{j}}, l, t}\right)}
$$

We exploit the binary labeling to evaluate the node's segmentation uncertainty as:

$$
U\left(\mathbf{c}_{\mathbf{j}}\right)=1-\left|P_{\text {seg }}\left(\mathbf{c}_{\mathbf{j}} \mid S\right)-0.5\right|
$$

Using this formulation, $U\left(\mathbf{c}_{\mathbf{j}}\right)$ is minimum when the optimal segmentation label is the most likely label, and maximum when both labels are equally probable.

If a node's segmentation is certain, it should be propagated, while an uncertain segmentation should not influence the decisions at the next level. The segmentation labels are propagated via an inter levels potential between the nodes $\mathbf{c}_{\mathbf{j}} \in \mathcal{G}_{j, \max }$ and $\mathbf{p} \in \mathcal{G}_{j+1, \text { max }}$ that are connected, that depends on the reliability of the neighbors' segmentation:

$$
V_{u c y}\left(l_{c_{j}}, l_{p}\right)= \begin{cases}0 & \text { if } s^{l_{c_{j}}}=s^{l_{p}} \\ -\log \left(U\left(\mathbf{c}_{\mathbf{j}}\right)\right) & \text { Otherwise }\end{cases}
$$

This potential penalizes the segmentation labels that are not in accordance with the lower resolution level's labeling. The penalty will be important if the segmentation decisions at level $j$ were highly reliable and low otherwise. 
The node activation criterion for segmentation relies on this inter levels potential by activating the nodes that are not strongly penalized or equally penalized for both labels:

$$
A_{s}(\mathbf{p})=H\left(\left|\sum_{\mathbf{c}_{\mathbf{j}} \in \mathcal{N}(\mathbf{p})} \frac{(-1)^{s^{l_{p}}}}{N} V_{u c y}\left(l_{c_{j}}, l_{p}\right)\right|-t s h\right)
$$

Where $N$ is the number of nodes in $\mathcal{N}(\mathbf{p}), H($.$) is the$ Heaviside function and $t s h$ is a threshold parameter. Each node in $\mathcal{G}_{j+1}$ can be connected to one or more nodes in $\mathcal{G}_{j}$. This term evaluates the mean segmentation confidence over all nodes connected to $\mathbf{p}$, taking into account the value of the segmentation label. It activates nodes whose neighbors' segmentations are uncertain or if the neighborhood consists of different labels that are equally confident (implying that the node is localized at a tumor's boundary).

Eventually, we rewrite the MRF energy at level $j$ and iteration $i$ :

$$
\begin{aligned}
& E_{\text {reg,seg }}\left(l \mid V, A \circ \mathcal{T}^{i-1}, \mathcal{G}_{j}\right)=\frac{1}{\left|\mathcal{G}_{j}\right|} \sum_{p \in \mathcal{G}} V_{p}\left(l_{p} \mid V, A \circ \mathcal{T}^{i-1}\right) \\
& +\sum_{p \in \mathcal{G}} \sum_{q \in \mathcal{N}(p)} V_{p q}\left(l_{p}, l_{q}\right)+\sum_{p \in \mathcal{G}_{j}} \sum_{c \in \mathcal{N}(p)} \frac{1}{N} V_{u c y}\left(l_{c_{j-1}}, l_{p_{j}}\right)
\end{aligned}
$$

where $\mathrm{N}$ is the number of nodes in $\mathcal{G}_{j-1}$ in the neighborhood of node $\mathbf{p} \in G_{j}$.

\section{Experimental Validation}

Our data set consisted of 102 3D MRI FLAIR volumes of patients featuring low grade gliomas. All volumes were manually segmented by experts. The volumes' sizes ranged from $256 \times 256 \times 24$ to $512 \times 512 \times 33$, and resolution from $0.4 \times 0.4$ to $0.9 \times 0.9 \mathrm{~mm}^{2}$ in the $(\mathrm{x}, \mathrm{y})$ plane and 5.3 to $6.4 \mathrm{~mm}$ in the $\mathrm{z}$ plane. The tumor size ranged from 3.5 $\mathrm{cm}^{3}$ to $230 \mathrm{~cm}^{3}$. The healthy brain template for registration was a 3D MRI FLAIR volume of size $256 \times 256 \times 24$, and resolution $0.9 \times 0.9 \times 5.45 \mathrm{~mm}^{3}$. As preprocessing, all images were skullstripped and their intensity was regularized. In order to preserve the contrast between tumor and background, we opted for a simple regularization method, where all images were set to the same median and interquartile range as the reference template. Prior to performing the deformable registration, all volumes were rigidly registered to the healthy template.

\subsection{Segmentation prior}

We constructed the segmentation probability prior using the Gentle adaboost algorithm [4]. 36 volumes were randomly selected from our data set in order to learn the tumor vs background classifier: to each voxel is associated a feature vector, constructed using intensity based features (patches around the voxel, entropy, standard deviation and median over patches of variable sizes), Gabor features [10] and asymmetry between the left and right hemispheres. The algorithm constructs a strong classifier as a linear combination of weak classifiers. Each classifier is a decision stump with respect to one of the features' value. When applied to a new volume, the strong classifier yields a score $S_{b}(\mathbf{x})$ that can be converted to a probability as:

$$
\begin{aligned}
p_{t m}(\mathbf{x}) & =\frac{1}{1-\exp \left(-2 S_{b}(\mathbf{x})\right)} \\
p_{b g}(\mathbf{x}) & =1-p_{t m}(\mathbf{x})
\end{aligned}
$$

\subsection{Implementation}

Our incremental approach consisted of 4 grid levels and 3 image levels where the resolution of the image increases with the grid's resolution. At the fourth grid level, the image's resolution remains the same. The grid's resolution was progressively refined from $9 \times 9 \times 5$ to $65 \times 65 \times 37$. We use cubic B-splines as a projection function. We progressively diminish the value of $\alpha$ from 1 to 0.015 , so that the presence of the tumor has an increasing impact on the registration, and the finest level focuses solely on segmentation. This enables to first focus on the registration of the brain's main structures, when the resolution of the grid and the image is low enough for the presence of the tumor to have a limited impact. This limits the false detections that would correspond to misaligned areas instead of tumorous areas. Inversely, the parameter $C_{t m}$ was progressively increased from 6 to 12 times the mean value of the similarity criterion. $\lambda$ was set to 20 and relaxed in the tumor area to allow for the strong displacements that can be caused by a tumor. The similarity criterion adopted was the Sum of Absolute Differences (SAD). The same set of parameters was used for all the volumes we tested.

We perform 3 iterations at each grid level. The local registration uncertainties are evaluated during the first iteration, where we adopt a dense displacement sampling, with 5 sampling steps in each direction (1331 labels). In the following level, we adopt a sparse sampling, the displacement labels being sampled along the uncertainty covariance axes (31 labels). This allows to exploit the uncertainty information with limited impact on the run time. In the last level, the displacement set is simply refined by reducing the maximum displacement, still sampled along the covariance's main axes. The maximum displacement is initially set to 0.4 times the grid spacing, in order to preserve a diffeomorphic transformation [2].

\subsection{Results}

We tested our method on the 66 remaining volumes. Fig.[5] shows the percentage of activated nodes with respect to the maximal resolution possible and visual exam- 

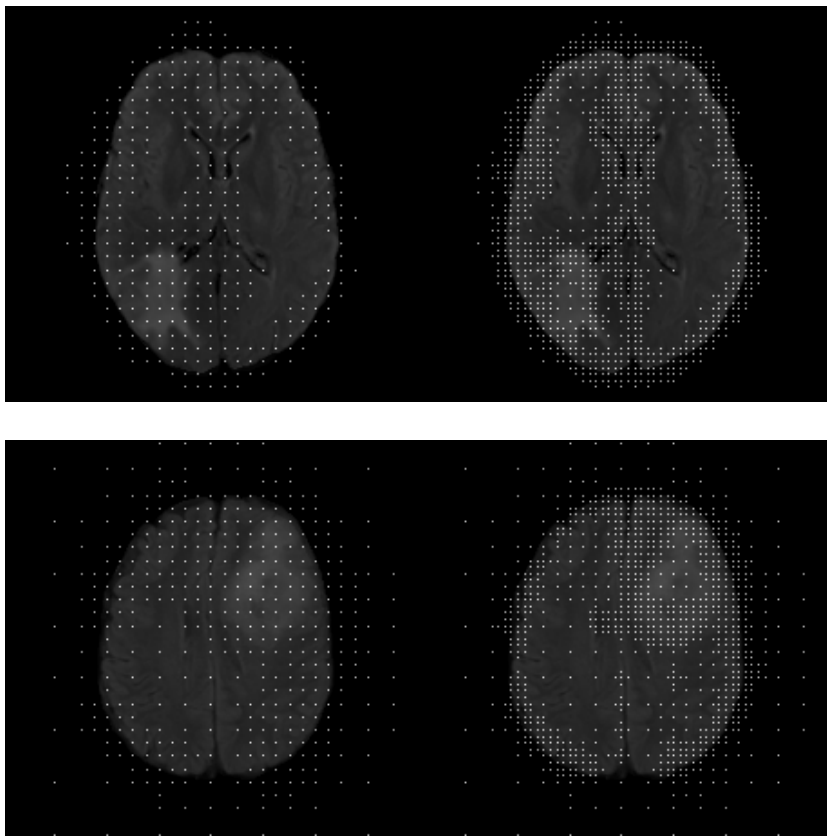

Figure 4: Visual examples of the activated nodes for the last 2 levels of the incremental approach. The nodes are superimposed to the target image.

ples are shown in Fig.[4]. Nodes are activated in the presence of brain structures and around the tumor boundary, and their number is significantly lower than the total number of potential nodes, strongly reducing the complexity of the framework. We compared our results to the joint segmentation and registration method [11] with the maximal grid resolution and a uniform grid of the same final resolution as the resolution obtained with adaptive resampling, using the best set of parameters and relaxing the registration pairwise cost in the tumor area. To evaluate the registration, the ventricles where segmented on 27 volumes that were affected by the presence of the tumor. The Dice score, false positive rate, true positive rate and mean absolute distance (MAD) between contours were evaluated with respect to the manual segmentations of the tumor and ventricles outside the tumor area. Fig.[6] shows boxplots of the different segmentation scores for the 3 different methods and registration results are shown in Fig.[7]. Our method shows equivalent results for both segmentation and registration with a much lower complexity ( $20 \%$ of activated nodes in the last level), while a uniform grid of the same complexity yields lower quality registrations and poorly detected tumors. Visual results are shown in Fig.[8], showing the strong correspondences between the source and deformed target image.

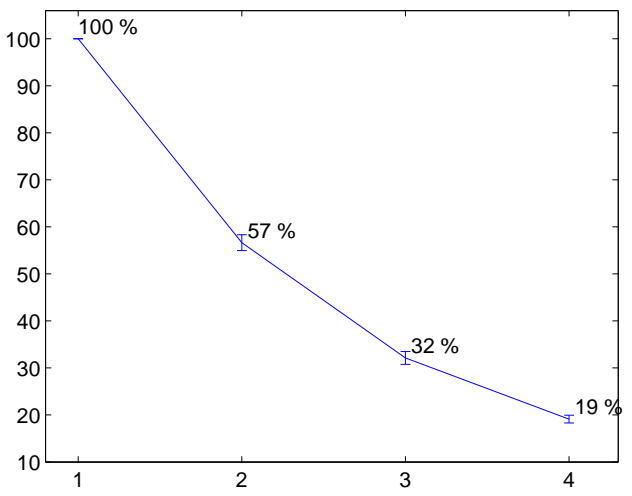

Figure 5: Mean percentage of activated nodes per level.

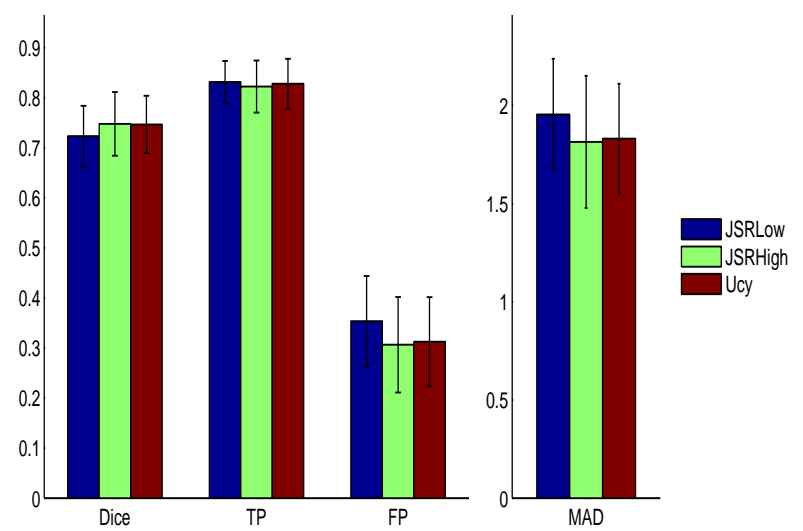

Figure 7: Registration results: Error bar graphs of the Dice, True Positives (TP), False Positives (TP) and MAD scores obtained for the method in [11] with low (left, JSRLow) and high resolution (middle, JSRHigh), and our method (right, Ucy)

\section{Discussion}

In this paper we have proposed a novel stochastic formulation for combined atlas registration and tumor segmentation where the obtained solutions are associated with covariance matrices efficiently determined through the graph marginals. The method explores the registration and segmentation uncertainties towards efficient sampling of the discrete deformation space and for the adaptive piece-wise regular refinement of the grid structure. The proposed formulation provides a statistical interpretation of the solution, while preliminary results demonstrate that the performance remains about the same while considering a much smaller content-adaptive finer resolution graph. Relying on sparse content adaptive grids can further improve the quality of the results by enabling to reach locally voxel level resolu- 


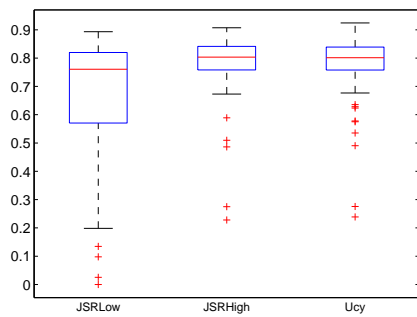

(a)

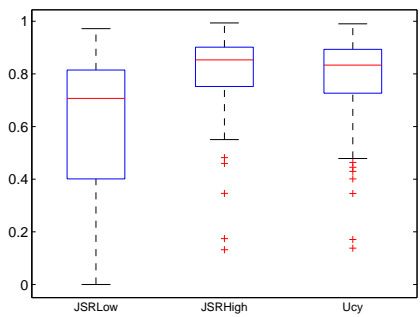

(b)

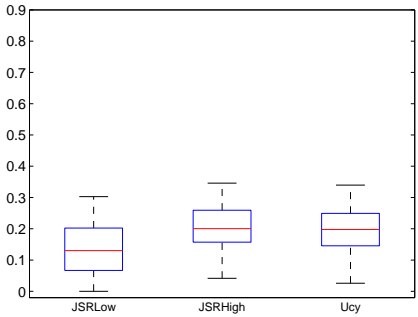

(c)

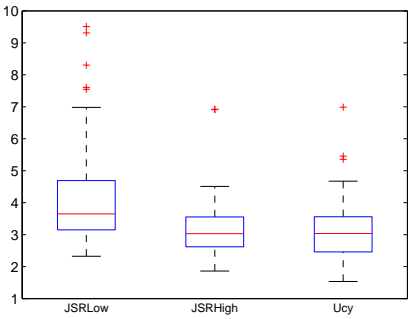

(d)

Figure 6: Segmentation results: Boxplots of the Dice score (a) True Positive (b) and False Positive (c) rates and MAD score (d) for the method in [11] with low (left, JSRLow) and high resolution (middle, JSRHigh), and our method (right, Ucy)

tions for precise segmentations in a computationally efficient manner.

Going beyond tumor segmentation, applying such a formulation to atlas-based segmentation is an interesting clinical perspective. The consideration of the expected stochastic nature of the solution from the very beginning and not as a by-product of the optimization procedure could be a significant theoretical break-through. In simple words the idea would be to introduce a graph-based objective function where the statistical nature of the expected solution is explicitly encoded and estimated during the inference process (through for example sampling of the covariance matrices along with the space of solutions). One could expect a better approximation of the statistical behavior of the problem as well as robustness since the objective function integrates supports from all possible solutions from a given distribution.

\section{References}

[1] M. Bach Cuadra, M. De Craene, V. Duay, B. Macq, C. Pollo, and J. Thiran. Dense deformation field estimation for atlasbased segmentation of pathological MR brain images. Computer methods and programs in biomedicine, 84(2-3):66-75, 2006. 1

[2] Y. Choi and S. Lee. Injectivity conditions of $2 d$ and $3 d$ uniform cubic b-spline functions. Graphical Models, 62:2000, 2000. 5

[3] A. Doucet, N. de Freitas, K. Murphy, and S. Russell. Raoblackwellised particle filtering for dynamic bayesian networks. In Proceedings of the Sixteenth Conference Annual Conference on Uncertainty in Artificial Intelligence (UAIO0), pages 176-183, 2000. 1

[4] J. Friedman, T. Hastie, and R. Tibshirani. Additive Logistic Regression: a Statistical View of Boosting. The Annals of Statistics, 38(2):337-407, 2000. 2, 5

[5] B. Glocker, N. Paragios, N. Komodakis, G. Tziritas, and N. Navab. Optical flow estimation with uncertainties through dynamic mrfs. In $C V P R, 2008.1$

[6] A. Gooya, K. M. Pohl, M. Bilello, G. Biros, and C. Davatzikos. Joint Segmentation and Deformable Registration of Brain Scans Guided by a Tumor Growth Model. In MICCAI, 2011. 1

[7] J. E. Iglesias, M. R. Sabuncu, and K. V. Leemput. Incorporating parameter uncertainty in bayesian segmentation models: Application to hippocampal subfield volumetry. In MICCAI, pages 50-57, 2012. 1

[8] P. Kohli and P. H. S. Torr. Measuring uncertainty in graph cut solutions. Comput. Vis. Image Underst., 112:30-38, 2008. 1, 3

[9] N. Komodakis, G. Tziritas, and N. Paragios. Performance vs computational efficiency for optimizing single and dynamic mrfs: Setting the state of the art with primal-dual strategies. Computer Vision and Image Understanding, 112(1):14-29, 2008. 2

[10] B. S. Manjunath and W.-Y. Ma. Texture features for browsing and retrieval of image data. IEEE TPAMI, 18(8):837842, 1996. 5

[11] S. Parisot, H. Duffau, S. Chemouny, and N. Paragios. Joint tumor segmentation and dense deformable registration of brain mr images. In MICCAI, pages 651-658, 2012. 1, 2, $3,6,7$

[12] M. Prastawa, E. Bullitt, S. Ho, and G. Gerig. A brain tumor segmentation framework based on outlier detection. Medical Image Analysis, 8(3):275-283, 2004. 1

[13] K. Rohr. Image registration based on thin-plate splines and local estimates of anisotropic landmark localization uncertainties. In MICCAI, pages 1174-1183, 1998. 1

[14] D. Rueckert, L. Sonoda, I. Hayes, D. Hill, M. Leach, and D. Hawkes. Nonrigid registration using free-form deformations: Application to breast $\mathrm{mr}$ images. IEEE TMI 1999, 18(8):712-721, 08 1999. 2

[15] W. Shi, X. Zhuang, L. Pizarro, W. Bai, H. Wang, K.-P. Tung, P. J. Edwards, and D. Rueckert. Registration using sparse free-form deformations. In MICCAI, pages 659-666, 2012. 1

[16] C. V. Stewart, Y.-L. Lee, and C.-L. Tsai. An uncertaintydriven hybrid of intensity-based and feature-based registration with application to retinal and lung ct images. In MICCAI, pages 870-877, 2004. 1

[17] M. Taron, N. Paragios, and M.-P. Jolly. Registration with uncertainties and statistical modeling of shapes with variable metric kernels. IEEE TPAMI, 31(1):99-113, 2009. 1 

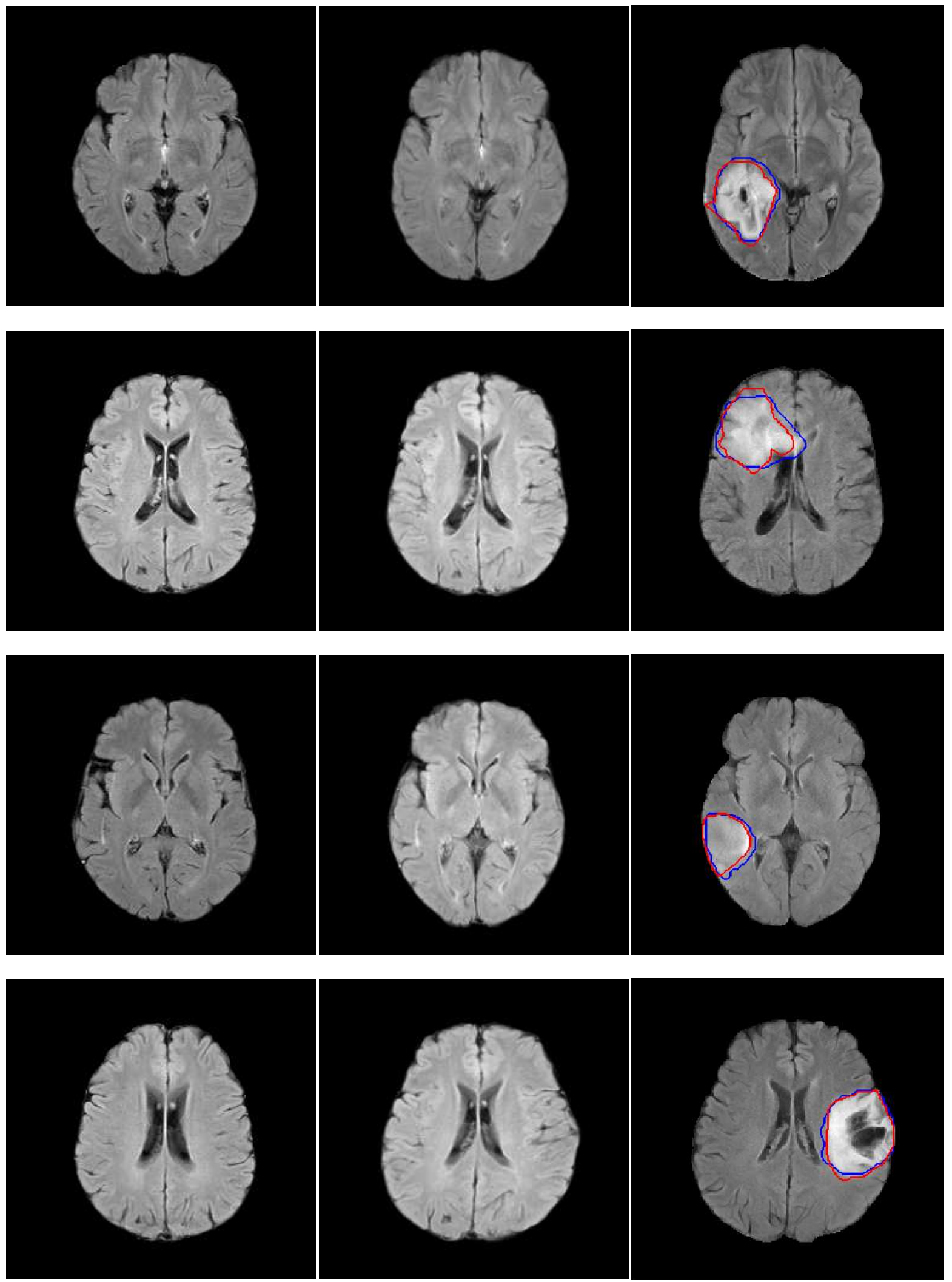

(a)

(b)

(c)

Figure 8: Visual Registration and Segmentation results. (a) Source image, (b) Deformed source image, (c) Target image segmented: Automatic Segmentation (blue) and Manual Segmentation (red) 